\title{
Impact of Regionalized Care on Concordance of Plan and Preventable Adverse Events on General Medicine Services
}

\author{
Stephanie K. Mueller, MD, MPH ${ }^{1,2 *}$, Jeffrey L. Schnipper, MD, MPH ${ }^{1,2}$, Kyla Giannelli, PA-C ${ }^{1}$, \\ Christopher L. Roy, MD ${ }^{1,2}$, Robert Boxer, MD, PhD ${ }^{1,2}$
}

${ }^{1}$ Brigham and Women's Hospital Hospitalist Service and Division of General Internal Medicine, Brigham and Women's Hospital, Boston, Massachusetts; '2Department of Medicine, Harvard Medical School, Boston, Massachusetts.

BACKGROUND: Dispersion of inpatient care teams across different medical units impedes effective team communication, potentially leading to adverse events (AEs).

OBJECTIVE: To regionalize 3 inpatient general medical teams to nursing units and examine the association with communication and preventable AEs.

DESIGN: Pre-post cohort analysis.

SETTING: A 700-bed academic medical center.

PATIENTS: General medicine patients on any of the participating nursing units before and after implementation of regionalized care.

INTERVENTION: Regionalizing 3 general medical physician teams to 3 corresponding nursing units.

MEASUREMENTS: Concordance of patient care plan between nurse and intern, and adjusted odds of preventable AEs.

RESULTS: Of the 414 included nurse and intern paired surveys, there were no significant differences pre- versus postregionalization in total mean concordance scores $(0.65$ vs $0.67, P=0.26)$, but there was significant improvement in agreement on expected discharge date (0.56 vs $0.68, P=$ $0.003)$, knowledge of the other provider's name (0.56 vs $0.86, P<0.001)$, and daily care plan discussions (0.73 vs 0.88, $P<0.001$ ). Of the 392 reviewed patient medical records, there was no significant difference in the adjusted odds of preventable AEs pre- versus postregionalization (adjusted odds ratio: 1.37, 95\% confidence interval: 0.69, 2.69).

CONCLUSIONS: We found that regionalization of care teams improved recognition of care team members, discussion of daily care plan, and agreement on estimated discharge date, but did not significantly improve nurse and physician concordance of the care plan or reduce the odds of preventable AEs. Our findings suggest that regionalization alone may be insufficient to effectively promote communication and lead to patient safety improvements. Journal of Hospital Medicine 2016;11:620-627. (C) 2016 Society of Hospital Medicine
Failures in communication among healthcare professionals are known threats to patient safety. These failures account for over $60 \%$ of root causes of "sentinel events," the most serious events reported to The Joint Commission. ${ }^{1}$ As such, identifying both patterns of effective communication as well as barriers to successful communication has been a focus of efforts aimed at improving patient safety. However, to date, the majority of this work has centered on improving communication in settings such as the operating room and intensive care unit, ${ }^{2-4}$ or at times of care transitions. ${ }^{5-8}$

Unique barriers exist for effective interdisciplinary communication in the hospital setting, particularly physician-nurse communication regarding shared hos-

\footnotetext{
*Address for correspondence and reprint requests: Stephanie Mueller MD, Division of General Internal Medicine, Brigham and Women's Hospital, 1620 Tremont Street, Roxbury, MA 02120; Telephone: 617-278-0628; Fax: 617-732-7072; E-mail: smueller1@partners.org

Additional Supporting Information may be found in the online version of this article.

Received: September 28, 2015; Revised: January 14, 2016; Accepted: January 28, 2016

2016 Society of Hospital Medicine DOI 10.1002/jhm.2566

Published online in Wiley Online Library (Wileyonlinelibrary.com).
}

pitalized patients. ${ }^{9}$ Traditionally, care of hospitalized patients is provided by physicians, nurses, and other team members working in varied workflow patterns, leading to dispersed team membership, where each team member cares for different groups of patients in different locations across the hospital. This dispersion is further heightened on teaching services, where residents' rotation schedules lead to frequent changes of care team membership, leaving inpatient care teams particularly vulnerable to ineffective communication. Evidence suggests that communication between nurses and physicians is currently suboptimal, leading to frequent disagreement regarding the patient's plan of care. ${ }^{9,10}$ This divergence between physician and nursing perceptions of patients' care plans may leave patients at greater risk of adverse events (AEs).

Several studies have examined the effects of regionalized inpatient care teams, where multidisciplinary team members care for the same patients on the same hospital unit, on communication and patient outcomes. ${ }^{4,11-14}$ Results of these studies have been inconsistent, perhaps due to the particular characteristics of the care teams or to the study methodology. Thus, further rigorously done studies are required to better 


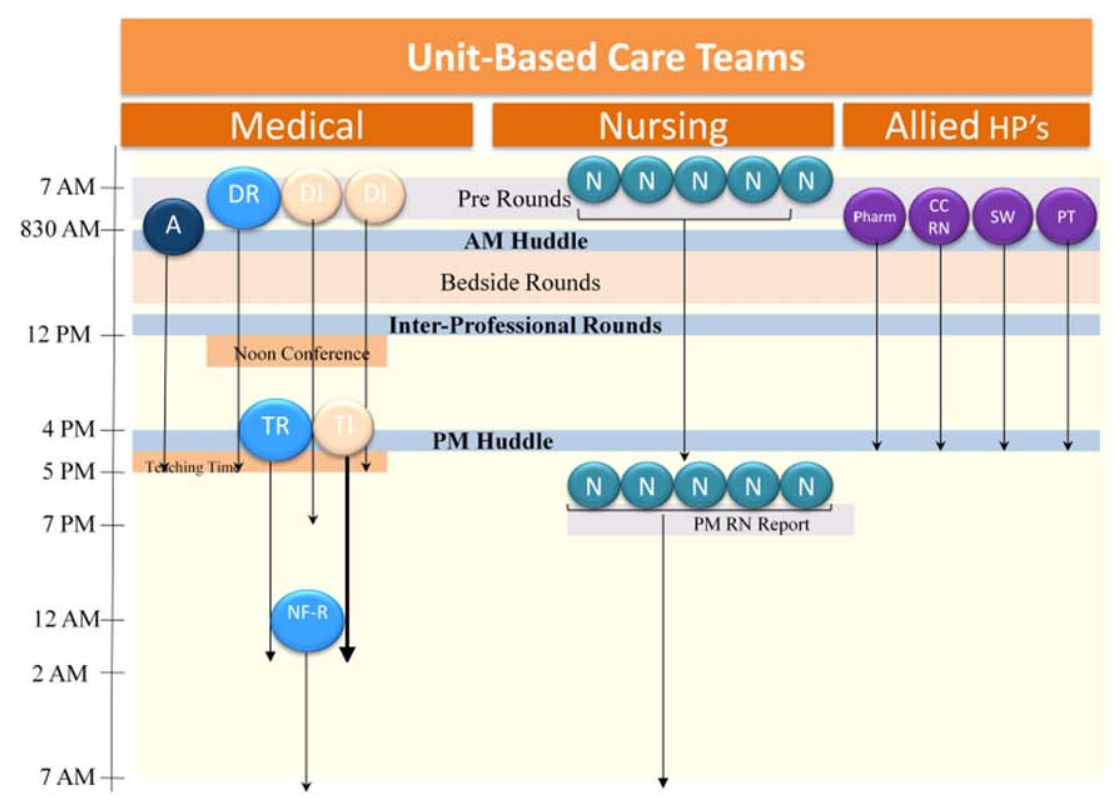

\begin{tabular}{|l|l|}
\hline \multicolumn{2}{|c|}{ AM Huddle } \\
(8:30-8:40am)
\end{tabular}

FIG. 1. Regionalization of general medical services into united-based care teams. Regionalization of general medical services involved included localizing each physician care team to a single nursing unit. Physician care teams included shared patient care responsibilities between a day team consisting of an attending hospitalist (A), a daytime resident (DR), and 2 daytime interns (DI), and a "twilight team" consisting of a twilight resident (TR) and twilight intern (TI), limiting hours of cross-coverage by a night-float resident (NF-R). In addition, structured interdisciplinary structured huddles were scheduled throughout the day to identify workflow needs (eg, calling interpreter prior to bedside rounds), create patient care plans, and anticipate patient discharges. This creates a virtuous cycle of shared responsibility between care team members to improve efficiency, create earlier bed availability, and improve regionalization. $\mathrm{Abbreviations:} \mathrm{AM}=\mathrm{Morning}, \mathrm{CC}=\mathrm{Care}$ Coordinator, $\mathrm{RN}=$ Nurse, $\mathrm{N}=$ Nurse, $\mathrm{OT}=$ Occupational Therapist, $\mathrm{PM}=$ Evening, $\mathrm{PT}=$ Physical Therapist, $\mathrm{SW}=$ Social Worker.

understand the impact of team regionalization on patient care. The goal of this study was to examine whether the implementation of regionalized inpatient care teams was associated with improvements in care team communication and preventable AEs.

\section{METHODS}

\section{Setting, Patients, and Study Design}

We performed a cohort analysis of patients at a 700bed tertiary care center, pre- and postregionalization of inpatient general medicine care teams. Our study protocol was approved by the Partners Healthcare Human Subjects Review Committee. Patients were eligible for inclusion if they were 18 years of age or older and discharged from the general medicine service (GMS) from any of the 3 participating nursing units between April 1, 2012 and June 19, 2012 (preregionalization) or April 1, 2013 and June 19, 2013 (postregionalization).

\section{Intervention}

On June 20, 2012, regionalized care was implemented on the GMS such that each of 3 GMS teams was localized to 1 of 3, 15-bed nursing units. Prior to regionalization, the GMS physician care teams, each consisting of 1 hospitalist attending, 1 medical 
resident, and 2 medical interns, would care for patients on an average of 7 and up to 13 different nursing units on a given day.

Regionalized care consisted of a multifaceted intervention codeveloped by hospitalist, residency, nursing, emergency department, and hospital leadership and included: (1) regionalizing GMS teams as much as possible; (2) change in resident call structure from a traditional 4-day call cycle to daily admitting; (3) collaborative efforts to enhance GMS patient discharges before noon to promote regionalized placement of patients without prolonging time in the emergency department (ED); (4) daily morning and postround multidisciplinary huddles to prioritize sicker patients and discharges; (5) encouragement of daily rounds at patients' bedsides with presence of physician team, nurse, and team pharmacist if available; (6) creation of unit- and team-level performance reports; and (7) creation of unit-based physician and nursing co-leadership (Figure 1). ${ }^{15}$

\section{Concordance of Plan}

Concordance of plan was measured via a 7-question survey previously developed, pilot tested, and used to measure the impact of regionalized care on care team communication between inpatient nurse-physician team members. ${ }^{9}$ The survey was administered inperson by 1 of 8 trained research assistants (RAs) (4/ intervention period) to nurse and intern pairs caring for patients on the study units pre- and postregionalization. GMS patients were eligible for inclusion if surveys could be administered to their nurse and intern within the first 24 hours of admission to the unit and within 48 hours of admission to the hospital, based on RA availability (thus excluding patients admitted on Fridays as surveys were not conducted over the weekend). Most often, all eligible patients admitted to the study units during time periods of data collection were included in the study. On limited occasions, the daily supply of patients surpassed RA capacity for inclusion, at which time computer-generated randomization was utilized to randomly select patients for inclusion. Nurse and intern pairs were surveyed once during a patient's hospitalization, although they could be surveyed more than once about different patients, and patients could be included more than once if rehospitalized on the study unit and cared for by a different nurse-intern pair. Of the 472 selected eligible patients, the nurses and interns of 418 patients were available and consented to survey administration, representing 361 unique nurse and intern pairs and 399 unique patients.

Each member of the pair was asked about 7 specific aspects of the patient's care plan for that day in isolation from the other team member, including: (1) the patient's primary diagnosis, (2) the patient's expressed chief concern, (3) the day's scheduled tests, (4) the day's scheduled procedures, (5) consulting services involved, (6) medication changes made that day, and (7) the patient's expected discharge date. In addition, each pair was asked the name of the other team member (ie, the nurse was asked the name of the intern and vice versa), and whether or not the patient care plan for the day had been discussed with the other team member, where concordance was defined as both members agreeing the plan had been discussed. All responses were recorded verbatim. Pairs were surveyed independently between 12 PM and 2 PM, limiting confounding by evolving plans of care over time.

Each set of surveys were then reviewed by 2 of 4 trained adjudicators, and responses to each question were scored as complete, partial, or no agreement. Rules for degree of agreement were based upon previously utilized parameters ${ }^{9}$ as well as biweekly meetings during which common themes and disagreements in ratings were discussed, and rules generated to create consensus (see Supporting Information, Appendix, in the online version of this article).

\section{Adverse Event Detection}

Of the patients meeting eligibility criteria, 200 patients were randomly selected using computergenerated randomization from each time period for $\mathrm{AE}$ outcome assessment, for a total of 400 patients.

Each patient's electronic medical record was retrospectively reviewed by a trained clinician using a previously validated screening tool to detect any possible AEs. ${ }^{11}$ Any positive screen prompted documentation of a narrative summary including a short description of the possible $\mathrm{AE}$ and pertinent associated data. We defined $\mathrm{AE}$ as any injury due to medical management rather than the natural history of the illness, and further limited this definition to only include AEs that occurred on the study unit or as a result of care on that unit.

Two of 4 trained adjudicators, blinded to time period, then separately reviewed each narrative summary using previously validated 6-point confidence scales to determine the presence and preventability of $\mathrm{AE}$, with confidence ratings of 4 or greater used as cutoffs. ${ }^{11}$ All AEs were also scored on a 4-point severity scale (trivial, clinically significant, serious, or life threatening), with severe $\mathrm{AE}$ defined as serious or life threatening. Lastly, adjudicators grouped AEs into 1 of 10 prespecified categories. ${ }^{11}$ Any disagreements in ratings or groupings were discussed by all 4 adjudicators to reach consensus.

\section{Data Analysis}

Patient characteristics are presented using descriptive statistics and were compared in the pre- and postregionalization time periods using $\chi^{2}$ or $t$ tests as appropriate.

To analyze whether regionalized care was associated with concordance of plan, adjudicated survey questions were assigned points of $1,0.5$, and 0 for 
TABLE 1. Baseline Characteristics

\begin{tabular}{|c|c|c|c|c|c|c|}
\hline \multirow[b]{2}{*}{ Characteristic } & \multicolumn{3}{|c|}{ Concordance of Care Plan } & \multicolumn{3}{|c|}{ Adverse Events } \\
\hline & Pre, $n=197$ & Post, $n=217$ & $P$ Value & Pre, $n=198$ & Post, $n=194$ & $P$ Value \\
\hline Age, mean (SD) & $60.5(19.4)$ & $57.6(20.8)$ & 0.15 & $60.4(18.9)$ & $58.0(21.2)$ & 0.24 \\
\hline Race/ethnicity, n (\%) & & & 0.34 & & & 0.12 \\
\hline White & $134(68.0)$ & $141(65.0)$ & & $132(66.5)$ & $121(62.4)$ & \\
\hline Black & $42(21.3)$ & $45(20.7)$ & & $41(20.8)$ & $54(27.8)$ & \\
\hline Language, $n(\%)$ & & & 0.30 & & & 0.73 \\
\hline English & $183(92.9)$ & $203(93.5)$ & & $176(88.7)$ & $175(90.2)$ & \\
\hline Spanish & $6(3.0)$ & $10(4.6)^{\prime}$ & & $10(5.2)$ & $10(5.3)^{\prime}$ & \\
\hline Other & $8(4.1)$ & $4(1.8)$ & & $12(6.1)$ & $9(4.5)$ & \\
\hline Admitting source, $n(\%)$ & & & 1.00 & & & 0.10 \\
\hline Physician office & $13(6.6)$ & $13(6.0)$ & & $13(6.6)$ & $6(3.1)$ & \\
\hline DRG weight, mean (SD) & $1.6(1.0)$ & $1.5(1.0)$ & 0.37 & $1.5(0.93)$ & $1.5(1.1)$ & 0.96 \\
\hline
\end{tabular}

NOTE: Abbreviations: DRG, diagnosis-related group; IQR, interquartile range; SD, standard deviation.

complete, partial, and no agreement, respectively. Total mean concordance scores for any patient ranged from 0 to 7 points, and were divided by total number of answered questions (up to 7) for a range of 0 to 1. Total mean concordance scores as well as mean concordance score per survey question were compared pre- versus postregionalization using $t$ tests. In sensitivity analyses, adjudicated survey responses were dichotomized with "complete" and "partial" agreement deemed concordant responses. Percent concordance for each question was then compared pre- versus postregionalization using $\chi^{2}$ analysis. Questions about the name of the other team member and discussion of daily care plan with the other team member were excluded from total concordance score calculations and were compared individually pre- versus postregionalization, because they are not directly about the plan of care.

To analyze the association of regionalization with odds of preventable AE, we performed multivariable logistic regression adjusted for patient age, sex, race, language, and Elixhauser comorbidity score, ${ }^{16}$ and utilized generalized estimating equations to account for clustering by hospital unit. Secondary outcomes included severe preventable AEs, nonpreventable AEs, and category of preventable AEs using similar methodology. Two-sided $P$ values $<0.05$ were considered significant, and SAS version 9.2 (SAS Institute Inc., Cary, NC) was used for all analyses.

\section{RESULTS}

The fidelity of the intervention in achieving its goal of regionalized care is discussed separately. ${ }^{15}$ Briefly, the intervention was successful at achieving $85 \%$ regional- ization by team (ie, average daily percentage of team's patients assigned to team's unit) and $87 \%$ regionalization by unit (ie, average daily percentage of unit's patients with assigned team) following implementation, compared to $20 \%$ regionalization by team and unit in the preintervention period. Importantly, the average daily census of physician care teams rose by $32 \%$, from a mean of 10.8 patients/physician care team preregionalization to a mean of 14.3 patients/ physician care team postregionalization.

\section{Concordance of Plan}

Of the 418 nurse and intern paired surveys, 4 surveys were excluded due to repeat surveys of the same patient during the same hospitalization, for a total of 197 distinct paired surveys preregionalization and 217 paired surveys postregionalization. There were no statistically significant differences in patients' age, sex, race, language, admission source, length of stay, Elixhauser comorbidity score and diagnosis-related group weight pre- versus postregionalization (Table 1).

Kappa scores for adjudications of concordance surveys (defined as both adjudicators scoring the same level of agreement (ie, both "complete" or "partial" agreement versus "no agreement") ranged from 0.69 to 0.95 , by question. There were no significant differences in total mean concordance scores in the care plan pre- versus postregionalization $(0.65$ vs $0.67, P$ $=0.26$ ) (Table 2). Similarly, there were no significant differences in mean concordance score for each survey question, except agreement on expected date of discharge $(0.56$ vs $0.68, P=0.003)$, knowledge of the other provider's name, and agreement that discussion of the daily plan had taken place with the other pair 
TABLE 2. Effect of Regionalized Care on Concordance of Care Plan between Primary Nurse and Responding Physician

\begin{tabular}{|c|c|c|c|}
\hline Concordance Outcome & Pre, $n=197$ & Post, $\mathrm{n}=217$ & $P$ Value \\
\hline \multicolumn{4}{|l|}{ Concordance score* } \\
\hline Total concordance score, mean (SD)† & $0.65(0.17)$ & $0.67(0.16)$ & 0.26 \\
\hline \multicolumn{4}{|l|}{ Subgroups } \\
\hline Diagnosis & $0.77(0.32)$ & $0.72(0.35)$ & 0.11 \\
\hline Patient's chief concern & $0.48(0.44)$ & $0.48(0.43)$ & 0.94 \\
\hline Tests today & $0.67(0.40)$ & $0.71(0.42)$ & 0.36 \\
\hline Procedures today & $0.93(0.25)$ & $0.92(0.25)$ & 0.71 \\
\hline Medication changes today & $0.56(0.44)$ & $0.59(0.43)$ & 0.54 \\
\hline Consulting services & $0.59(0.44)$ & $0.60(0.44)$ & 0.82 \\
\hline Expected discharge date & $0.56(0.44)$ & $0.68(0.38)$ & 0.003 \\
\hline Responding clinician knowledge of nurse's name & $0.56(0.50)$ & $0.86(0.35)$ & $<0.001$ \\
\hline Nurse's knowledge of responding clinician's name & $0.56(0.50)$ & $0.88(0.33)$ & $<0.001$ \\
\hline Plan discussed & $0.73(0.45)$ & $0.88(0.32)$ & $<0.001$ \\
\hline \multicolumn{4}{|l|}{ Percent concordance, mean (SD)† } \\
\hline Diagnosis & $92.0(27.3)$ & $88.6(31.9)$ & 0.25 \\
\hline Patient's chief concern & $59.6(49.1)$ & $60.6(49.0)$ & 0.84 \\
\hline Tests today & $78.9(40.9)$ & $77.2(42.1)$ & 0.67 \\
\hline Procedures today & $93.5(24.8)$ & $94.1(23.7)$ & 0.80 \\
\hline Medication changes today & $66.3(33.6)$ & $69.9(46.0)$ & 0.44 \\
\hline Consulting services & $69.3(46.2)$ & $68.9(46.4)$ & 0.93 \\
\hline Expected discharge date & $67.5(47.0)$ & $82.6(38.0)$ & $<0.001$ \\
\hline Responding clinician knowledge of nurse's name & $55.7(49.8)$ & $85.6(35.2)$ & $<0.001$ \\
\hline Nurse's knowledge of responding clinician's name & $55.9(49.8)$ & $87.9(32.8)$ & $<0.001$ \\
\hline Plan discussed & $72.9(44.6)$ & $88.2(32.3)$ & $<0.001$ \\
\hline
\end{tabular}

NOTE: Abbreviations: SD, standard deviation. ${ }^{*}$ Calculation of concordance score: agree $=1$ point, partial agreement $=0.5$ points, disagree $=0$ points. $†$ Total concordance score excluded the following survey question responses: knowledge of other team member name and plan discussed. ‡Concordance defined as "agree" or "partial agreement." For responding clinician knowledge of nurse's name, nurse's knowledge of responding clinician's name, and plan discussed, all paired survey responses were either agree (1) or disagree (0).

member. Similar results were seen when results were dichotomized (ie, partial or complete agreement vs no agreement) (Table 2).

\section{Adverse Events}

Of the 400 patients screened for AEs, 8 were excluded due to missing medical record number (5) and discharge outside of study period (3). Of the final 392 patient screens (198 pre, 194 post), there were no significant differences in patients' age, sex, race, language, length of stay, or Elixhauser score pre- versus postregionalization (Table 1).

Kappa scores for adjudicator agreement were 0.35 for presence of $\mathrm{AE}$ and 0.34 for preventability of $\mathrm{AE}$. Of the 392 reviewed patient records, there were 133 total AEs detected (66 pre, 67 post), 27 preventable AEs (13 pre, 14 post), and 9 severe preventable AEs (4 pre, 5 post) (Table 3$)$. There was no significant difference in the adjusted odds of preventable AEs postversus preregionalization (adjusted odds ratio: 1.37, $95 \%$ confidence interval: $0.69,2.69)$. Although the low number of AEs rated as severe or life threatening precluded adjusted analysis, unadjusted results similarly demonstrated no difference in odds of severe preventable AEs pre- versus postregionalization. As expected, there was no significant difference in adjusted odds of nonpreventable AE after implementation of regionalized care (Table 3).
Similarly, there were no significant differences in category of preventable AE pre- versus postregionalization. The most frequent preventable AEs in both time periods were those related to adverse drug events and to manifestations of poor glycemic control, examples of which are illustrated (Table 4).

\section{DISCUSSION}

In this study of general medicine patients at a large academic medical center, we found that regionalization of care teams on general medicine services was associated with improved recognition of care team members and agreement on estimated date of patient discharge, but was not associated with improvement in overall nurse and physician concordance of the patient care plan, or the odds of preventable AEs.

This intervention importantly addresses the barrier of dispersion of team membership, a well-recognized barrier to interdisciplinary collaboration, ${ }^{17,18}$ particularly with resident physician teams due to frequently changing team membership. Localization of all team members, in addition to encouragement of daily collaborative bedside rounds as part of the regionalization initiative, likely contributed to our observed improvement in team member identification and discussion of daily care plans. Similarly, regionalization resulted in improved agreement in estimations of date of patient discharge. Focus on early patient discharges was an integral part of the implementation efforts; we 
TABLE 3. Adjusted Effect of Regionalization on Adverse Events*

\begin{tabular}{lccc}
\hline & \multicolumn{2}{c}{ No. of Adverse Events } & Adjusted Odds Ratio Post vs Pre (95\% Cl) \\
\cline { 2 - 3 } \multicolumn{1}{c}{ Adverse Events } & Pre, $\mathrm{n}=198$ & Post, $\mathrm{n}=194$ & $1.37(0.69,2.69)$ \\
Preventable & 13 & 14 & $-\dagger$ \\
Serious and preventable & 4 & 5 & $1.20(0.85,1.75)$ \\
Nonpreventable & 47 & 50 & \\
\hline
\end{tabular}

NOTE: Abbreviations: $\mathrm{Cl}$, confidence interval. ${ }^{*}$ Adjusted for patient age, sex, race, language, and comorbidity as measured by the Elixhauser score. ${ }^{\dagger}$ Number of events precluded adjusted analysis. Unadjusted odds ratio $=1.30$ $(0.34,4.91)$.

\begin{tabular}{|c|c|}
\hline \multicolumn{2}{|c|}{$\begin{array}{l}\text { TABLE 4. Examples of Preventable Adverse Events } \\
\text { Due to Adverse Drug Events and Manifestations of } \\
\text { Poor Glycemic Control }\end{array}$} \\
\hline Adverse drug event & $\begin{array}{l}\text { 29-year-old male with history of alcohol abuse, complicated by } \\
\text { prior withdrawal seizures/intensive care unit admissions, } \\
\text { presented with alcohol withdrawal. Started on standing and } \\
\text { PRN lorazepam, kept on home medications including stand- } \\
\text { ing clonidine, gabapentin, citalopram, quetiapine. Became } \\
\text { somnolent due to polypharmacy, utimately discontinued } \\
\text { quetiapine as discovered took only as needed at home for } \\
\text { insomnia }\end{array}$ \\
\hline $\begin{array}{l}\text { Manifestations of } \\
\text { poor glycemic } \\
\text { control }\end{array}$ & $\begin{array}{l}\text { 78-year-old male with recently diagnosed lymphoma, distant } \\
\text { history of bladder and prostate cancer status post ileal loop } \\
\text { diversion, presented status post syncopal event; during } \\
\text { event, spilled boiling water on himself leading to second- } \\
\text { degree burns on } 3 \% \text { of his body. Initially admitted to traumal } \\
\text { burn service, ultimately transferred to medical service for } \\
\text { ongoing multiple medical issues including obstructive urop- } \\
\text { athy, acute on chronic renal failure. Adverse event was } \\
\text { hyperglycemia (>350 mg/dL on }>2 \text { consecutive readings) in } \\
\text { the setting of holding his home insulin detemir and insulin } \\
\text { aspart (had been placed on insulin aspart sliding scale } \\
\text { alone). After hyperglycemic episodes, was placed back on } \\
\text { weight-based basal/nutritional insulin }\end{array}$ \\
\hline
\end{tabular}

NOTE: Abbreviations: PNR, pro re nata (as needed).

therefore hypothesize that mutual focus on discharge planning by both nurses and responding clinicians may have explained this observed result.

On the other hand, regionalization did not appreciably improve the overall concordance of care plan between nurses and interns, despite a significant increase in team members agreeing that the plan had been discussed. Our findings support similar prior research demonstrating that regionalizing hospitalist attendings to single nursing units had limited impact on agreement of care plan between physicians and nurses. ${ }^{13}$ Similarly, in settings where physicians and nurses are inherently regionalized, such as the intensive care unit ${ }^{4}$ or the operating room, ${ }^{3}$ communication between physicians and nurses remains difficult. Collectively, our findings suggest that colocalization of physicians and nurses alone is likely insufficient to improve measured communication between care team members. Existing literature suggests that more standardized approaches to improve communication, such as structured communication tools used during daily inpatient care ${ }^{19,20}$ or formalized team training, ${ }^{21-23}$ lead to improvements in communication and collaboration. Despite these findings, it is important to highlight that this study did not assess other measures of workplace culture, such as teamwork and care team cohesiveness, which may have been positively affected by this intervention, even without measurable effect on concordance of care plan. Additionally, as noted, the average daily census on each team increased by almost a third postintervention, which may have impeded improvements in care team communication.

In addition, we found that our intervention had no significant impact on preventable AEs or severe preventable AEs. Although we cannot exclude the possibility that more subtle AEs were missed with our methodology, our results indicate that regionalized care alone may be inadequate to improve major patient safety outcomes. As discussed, the volume of patients did increase postintervention; thus, another way to state our results is that we were able to increase the daily volume of patients without any significant decreases in patient safety. Nevertheless, the results on patient safety were less than desired. A recent review of interdisciplinary team care interventions on general medical wards similarly demonstrated underwhelming improvements in patient safety outcomes, although the reviewed interventions did not specifically address preventable AEs, a gap in the literature commented on by the authors. ${ }^{24}$ Other albeit limited literature has demonstrated improvement in patient safety outcomes via multifaceted efforts aimed at improving care team member communication. Notably, these efforts include colocalization of care team members to single units but also involve additional measures to improve communication and collaboration between care team members, such as structured communication during interdisciplinary rounds, and certification of key interdisciplinary teamwork skills. ${ }^{11,14}$ Although our regionalized care intervention included many similar features to these "accountable care units" (ACUs) including unit-based care teams, unit-level performance reporting, and unit-based physician and nursing coleadership, significant differences existed. Notably, in addition to the above features, the ACU model also incorporated highly structured communication models for interdisciplinary rounding, and certification processes to ensure an appropriate communication skill base 
among care team members. ${ }^{14}$ Thus, although creation of regionalized care teams is likely a necessary precursor to implementation of these additional measures, alone it may be insufficient to improve patient safety outcomes.

Importantly, in our study we identified that adverse drug events and manifestations of poor glycemic control occurred in high frequency both before and following implementation of regionalized care, supporting other literature that describes the prevalence of these AEs. ${ }^{11,25-27}$ These results suggest that targeted interventions to address these specific AEs are likely necessary. Notably, the intervention units in our study did not consistently employ clinical pharmacists assigned specifically to that unit's care team to allow for integration within the care team. As prior research has suggested that greater collaboration with clinical pharmacists results in reduction of adverse drug events, ${ }^{28}$ next steps may include improved integration of team-based pharmacists into the activities of the regionalized care teams. Inpatient management of diabetes also requires specific interventions, ${ }^{29-31}$ only some of which may be addressable by having regionalized care and better interdisciplinary communication.

Our findings are subject to several limitations. First, this was a single-site study and thus our findings may not be generalizable to other institutions. However, regionalized care is increasingly encouraged to optimize communication between care team members. ${ }^{17,18}$ Therefore, our null findings may be pertinent to other institutions looking to improve patient safety outcomes, demonstrating that additional initiatives will likely be required. Second, our modes of outcome measurement possess limitations. In measuring concordance of care plan, although previously used survey techniques were employed, ${ }^{9}$ the concordance survey has not been formally validated, and we believe some of the questions may have led to ambiguity on the part of the responders that may have resulted in less accurate responses, thus biasing toward the null. Similarly, in measuring AEs, the screening tool relied on retrospective chart review looking for specific AE types ${ }^{11}$ and thus may not have captured more subtle AEs. Additionally, our study may have been underpowered to demonstrate significant reduction in preventable AEs, although other studies of similar methodology demonstrated significant results with similar sample size. ${ }^{11}$ This was due in part to our lower-than-expected baseline $\mathrm{AE}$ rate (6.6\% compared with approximately $10.3 \%$ in previous studies). ${ }^{11}$ Lastly, our study solely examined the association of regionalization with concordance of care plan and preventable AEs, but importantly excluded other clinically important outcomes that may have been positively (or negatively) impacted by these regionalization efforts, such as ED wait times, provider efficiency (eg, fewer pages, less time in transit, more time at the bedside), interdisciplinary teamwork, or patient or provider satisfaction.

\section{CONCLUSION}

In summary, our findings suggest that regionalized care teams alone may be insufficient to effectively promote communication between care team members regarding the care plan or to lead to improvements in patient safety, although we recognize that there may have been benefits (or unintended harms) not measured in this study but are nonetheless important for clinical care and workplace culture. This is an important lesson, as many hospitals move toward regionalized care in an effort to improve patient safety outcomes. However, strengthening the infrastructure by colocalizing care team members to maximize opportunity for communication is likely a necessary first step toward facilitating implementation of additional initiatives that may lead to more robust patient safety improvements, such as structured interdisciplinary bedside rounds (eg, facilitating and training all team members to fulfill specific roles), teamwork training, and certification of key interdisciplinary teamwork skills. Additionally, close examination of identified prevalent and preventable AEs can help to determine which additional initiatives are most likely to have greatest impact in improving patient safety.

Disclosures: This research was supported by funds provided by Brigham and Women's Hospital (BWH) and by funds provided by the Department of Medicine at BWH. All authors had full access to all of the data in the study and were integrally involved in the design, implementation, data collection, and analyses. The first author, Dr. Stephanie Mueller, takes responsibility for the integrity for the data and the accuracy of the data analysis. Dr. Schnipper reports grants from Sanofi Aventis, outside the submitted work.

\section{References}

1. Joint Commission on Accreditation of Healthcare Organizations. Understanding and Preventing Sentinel Events in Your Health Care Organization. Oak Brook, IL: Joint Commission; 2008.

2. Lingard L, Espin S, Whyte S, et al. Communication failures in the operating room: an observational classification of recurrent types and effects. Qual Saf Health Care. 2004;13(5):330-334.

3. Makary MA, Sexton JB, Freischlag JA, et al. Operating room teamwork among physicians and nurses: teamwork in the eye of the beholder. J Am Coll Surg. 2006;202(5):746-752.

4. Thomas EJ, Sexton JB, Helmreich RL. Discrepant attitudes about teamwork among critical care nurses and physicians. Crit Care Med. 2003;31(3):956-959.

5. Arora V, Johnson J, Lovinger D, Humphrey HJ, Meltzer DO. Communication failures in patient sign-out and suggestions for improvement: a critical incident analysis. Qual Saf Health Care. 2005;14(6): 401-407.

6. Starmer AJ, Spector ND, Srivastava R, et al. Changes in medical errors after implementation of a handoff program. N Engl J Med. 2014; 371(19):1803-1812.

7. Gandara E, Moniz T, Ungar J, et al. Communication and information deficits in patients discharged to rehabilitation facilities: an evaluation of five acute care hospitals. J Hosp Med. 2009;4(8):E28-E33.

8. Kripalani S, LeFevre F, Phillips CO, Williams MV, Basaviah P, Baker DW. Deficits in communication and information transfer between hospital-based and primary care physicians: implications for patient safety and continuity of care. JAMA. 2007;297(8):831-841.

9. O'Leary KJ, Thompson JA, Landler MP, et al. Patterns of nursephysician communication and agreement on the plan of care. Qual Saf Health Care. 2010;19(3):195-199.

10. Evanoff B, Potter P, Wolf L, Grayson D, Dunagan C, Boxerman S. Can we talk? Priorities for patient care differed among health care providers. In: Henriksen K, Battles JB, Marks ES, Lewin DI, eds. 
Advances in Patient Safety: From Research to Implementation. Vol 1. Rockville, MD: Agency for Healthcare Research and Quality; 2005.

11. O'Leary KJ, Buck R, Fligiel HM, et al. Structured interdisciplinary rounds in a medical teaching unit: improving patient safety. Arch Intern Med. 2011;171(7):678-684.

12. O'Leary KJ, Haviley C, Slade ME, Shah HM, Lee J, Williams MV. Improving teamwork: impact of structured interdisciplinary rounds on a hospitalist unit. J Hosp Med. 2011;6(2):88-93.

13. O'Leary KJ, Wayne DB, Landler MP, et al. Impact of localizing physicians to hospital units on nurse-physician communication and agreement on the plan of care. J Gen Intern Med. 2009;24(11):1223-1227.

14. Stein J, Payne C, Methvin A, et al. Reorganizing a hospital ward as an accountable care unit. J Hosp Med. 2015;10(1):36-40.

15. Boxer R, Vitale M, Gershanik E, et al. 5th time's a charm: creation of unit-based care teams in a high occupancy hospital [abstract]. J Hosp Med. 2015;10 (suppl. 2). Available at: http://www.shmabstracts.com/ abstract/5th-times-a-charm-creation-of-unit-based-care-teams-in-ahigh-occupancy-hospital. Accessed July 28, 2015.

16. Elixhauser A, Steiner C, Harris DR, Coffey RM. Comorbidity measures for use with administrative data. Med Care. 1998;36(1):8-27.

17. O'Leary KJ, Ritter CD, Wheeler H, Szekendi MK, Brinton TS, Williams MV. Teamwork on inpatient medical units: assessing attitudes and barriers. Qual Saf Health Care. 2010;19(2):117-121.

18. Aronson MD, Neeman N, Carbo A, et al. A model for quality improvement programs in academic departments of medicine. Am J Med. 2008;121(10):922-929.

19. Narasimhan M, Eisen LA, Mahoney CD, Acerra FL, Rosen MJ. Improving nurse-physician communication and satisfaction in the intensive care unit with a daily goals worksheet. Am J Crit Care. 2006;15(2):217-222.

20. Pronovost P, Berenholtz S, Dorman T, Lipsett PA, Simmonds T, Haraden C. Improving communication in the ICU using daily goals. J Crit Care. 2003;18(2):71-75.

21. Haller G, Garnerin P, Morales MA, et al. Effect of crew resource management training in a multidisciplinary obstetrical setting. Int J Qual Health Care. 2008;20(4):254-263.
22. Morey JC, Simon R, Jay GD, et al. Error reduction and performance improvement in the emergency department through formal teamwork training: evaluation results of the MedTeams project. Health Serv Res. 2002;37(6):1553-1581.

23. Nielsen PE, Goldman MB, Mann S, et al. Effects of teamwork training on adverse outcomes and process of care in labor and delivery: a randomized controlled trial. Obstet Gynecol. 2007;109(1):48-55.

24. Pannick S, Davis R, Ashrafian H, et al. Effects of interdisciplinary team care interventions on general medical wards: a systematic review. JAMA Intern Med. 2015;175(8):1288-1298.

25. Bates DW, Miller EB, Cullen DJ, et al. Patient risk factors for adverse drug events in hospitalized patients. ADE Prevention Study Group. Arch Intern Med. 1999;159(21):2553-2560.

26. Kripalani S, Roumie CL, Dalal AK, et al. Effect of a pharmacist intervention on clinically important medication errors after hospital discharge: a randomized trial. Ann Intern Med. 2012;157(1): 1-10.

27. Donihi AC, DiNardo MM, DeVita MA, Korytkowski MT. Use of a standardized protocol to decrease medication errors and adverse events related to sliding scale insulin. Qual Saf Health Care. 2006; 15(2):89-91.

28. Kaboli PJ, Hoth AB, McClimon BJ, Schnipper JL. Clinical pharmacists and inpatient medical care: a systematic review. Arch Intern Med. 2006;166(9):955-964.

29. Maynard G, Lee J, Phillips G, Fink E, Renvall M. Improved inpatient use of basal insulin, reduced hypoglycemia, and improved glycemic control: effect of structured subcutaneous insulin orders and an insulin management algorithm. J Hosp Med. 2009;4(1):3-15.

30. Schnipper JL, Liang CL, Ndumele CD, Pendergrass ML. Effects of a computerized order set on the inpatient management of hyperglycemia: a cluster-randomized controlled trial. Endocr Pract. 2010;16(2): 209-218.

31. Schnipper JL, Ndumele CD, Liang CL, Pendergrass ML. Effects of a subcutaneous insulin protocol, clinical education, and computerized order set on the quality of inpatient management of hyperglycemia: results of a clinical trial. J Hosp Med. 2009;4(1):16-27. 